\title{
Inventário de Medos III: Avaliação das Qualidades Psicométricas em uma Amostra Portuguesa
}

\author{
Fear Survey Schedule-III: Evaluation of the Psychometric Properties \\ in a Portuguese Sample
}

\author{
Filomena Valadão Dias*, Isabel Leal \& João Marôco \\ Instituto Superior de Psicologia Aplicada, Lisboa, Portugal
}

\begin{abstract}
Resumo
Este estudo teve como objetivo avaliar as qualidades psicométricas do Fear Survey Schedule-III, em uma amostra portuguesa. Participaram 1.980 sujeitos selecionados por conveniência a partir de uma população adulta normativa. As idades dos participantes estavam compreendidas entre os 18 e os 80 anos $(M=39,5, D P=8,5)$, sendo $59 \%$ do sexo feminino. As qualidades psicométricas da escala foram avaliadas em suas facetas de sensibilidade psicométrica, validade de construto e confiabilidade. A validade externa de construto foi avaliada com análise multigrupos em amostra aleatória e independente da amostra de validação inicial. O modelo fatorial original proposto apresentou um ajustamento inaceitável à amostra de validação. Procedeu-se ao refinamento do modelo de medida em uma parte da amostra, selecionada aleatoriamente. Em conclusão, o modelo de medida simplificado apresentou uma boa qualidade de ajustamento fatorial e foi invariante em uma segunda amostra independente da primeira. Propôs-se uma nova estrutura hierárquica, com fator de $2^{\mathrm{a}}$ ordem designado por "Medos", que revelou boas qualidades psicométricas (sensibilidade, validade de construto e confiabilidade). Palavras-chave: Medos, validade fatorial, confiabilidade, população normativa adulta.
\end{abstract}

\begin{abstract}
This study aimed at evaluating the psychometric properties of the Fear Survey Schedule-III in a Portuguese sample. A total 1,980 subjects, selected by convenience sampling from a normative adult population, participated in this study. The participants are aged between 18 and 80 years $(M=39.5$, $S D=8.5)$ and $59 \%$ of them are female. The psychometric properties of the scale were evaluated regarding psychometric sensitivity, construct validity, and reliability. The external construct validity was evaluated with multigroup analysis in a random sample, independent from the initial validation sample. The originally proposed factor model presented an unacceptable adjustment to the validation sample. Thus, it was proceeded the refinement of the model with a part of the sample which was randomly selected. In conclusion, the simplified model presented as a good factorial goodness of fit, and was invariant in a second sample, independent from the first. A new hierarchical structure was proposed with a $2^{\text {nd }}$ order factor called "Fears" which showed good psychometric properties (sensitivity, construct validity and reliability).

Keywords: Fears, factorial validity, reliability, normative adult population.
\end{abstract}

O medo é, geralmente, uma emoção comum sendo difícil imaginar a vida sem ele (Rachman, 2004). O medo saudável tem como função preparar e capacitar o indivíduo para lutar ou fugir perante o perigo percebido, sendo experienciado pela excitação fisiológica (como por exemplo, tensão muscular, suor e boca seca), pela cognição (por exemplo, pensamentos sobre morrer ou estar em perigo), e pelos comportamentos adotados (por exemplo, evitamento de situações temidas, fuga e luta) (Power \& Dalgleish, 2008).

* Endereço para correspondência: Instituto Superior de Psicologia Aplicada, Unidade de Investigação em Psicologia e Saúde, Rua Jardim do Tabaco, 34, Lisboa, Portugal 1149-041. E-mail: fdias@ispa.pt, isabel.leal@ iol.pt e jpmaroco@ispa.pt
Os conceitos de medo e ansiedade são descritos na literatura de forma pouco clara, sendo a definição dos seus limites controversa (Sylvers, Lilienfeld, \& LaPrairie, 2011). Segundo Öhman (2008) o medo e a ansiedade são estados aversivos sobrepostos, centrados na ameaça. Uma ameaça externa real pode despertar uma resposta de medo biologicamente apropriada, sendo considerado um medo adequado, se o indivíduo reage como se estivesse perante um perigo real, na ausência deste, o medo é considerado ansiedade (Dixon, de Monchaux, \& Sandler, 1957). Neste sentido a ansiedade é uma emoção relacionada com o comportamento de avaliação de riscos evocado em situações de perigo incerto, seja porque é uma nova situação ou porque o estímulo perigoso esteve presente no passado. O medo, por sua vez, está relacionado a estratégias defensivas em resposta a um perigo real (Blanchard 
\& Blanchard, 1988; Rosen \& Schulkin, 1998). Assim, o medo distingue-se da ansiedade essencialmente pela presença ou ausência de estímulos desencadeadores externos evidentes, e pelo comportamento de evitação do perigo. $\mathrm{O}$ medo pressupõe a existência de um estímulo desencadeador externo óbvio, que provoca comportamento de fuga ou evitação, enquanto a ansiedade é o estado emocional aversivo sem desencadeadores externos evidentes (Baptista, Carvalho, \& Lory, 2005; Öhman, 2008). De forma sucinta a ansiedade é conceptualizada como um estado em que o indivíduo é incapaz de ativar comportamentos claros e específicos no sentido de eliminar ou alterar o acontecimento/objeto/interpretação que está subjacente à dificuldade de concretização de um determinado objetivo (Power \& Dalgleish, 2008).

O medo e a ansiedade podem tornar-se uma perturbação pela experiência de um medo excessivo relativamente a objetos inofensivos, ou pelo desenvolvimento de crenças, de que algo é ameaçador ou prejudicial, quando efetivamente não é (Power \& Dalgleish, 2008; Rosen \& Schulkin, 1998). Atualmente, os vários sintomas das fobias e ansiedade patológica são descritos na abrangente categoria de perturbação de ansiedade apresentada na nomenclatura do Manual de diagnóstico e estatística das Perturbações Mentais (American Psychiatric Association, 2002). Estas perturbações têm um impacto significativo nas capacidades de funcionamento do indivíduo, no seu quotidiano, atuando de acordo com a sua cronicidade e severidade, no desenvolvimento de problemas físicos (por exemplo, doenças cardiovasculares); psicológicos (por exemplo, agorafobia) e sociais (por exemplo, evitamento social) (Baptista et al., 2005; Craske, 1977; Dixon et al., 1957; Rosen \& Schulkin, 1998; Sylvers et al., 2011; Taylor, 1998).

Para avaliação dos medos e sua intensidade, torna-se imprescindível a utilização de instrumentos de rastreamento/diagnóstico com adequadas características psicométricas. O inventário Fear Survey Schedule tem sido amplamente utilizado na análise dos medos e sua intensidade, quer em contextos clínicos como na investigação (de Jongh, Oosterink, Kieffer, Hoogstraten, \& Aartman, 2011; Tasto, 1977). O seu desenvolvimento tem-se dado ao longo dos anos. Hersen (1973) e Tasto (1977) apresentam um levantamento dos estudos realizados com o FSS desde 1956, descrevendo as várias versões, sua constituição e as diversas análises fatoriais realizadas. A versão mais utilizada nas últimas décadas é o FSS III, contudo, não existe consenso na literatura quanto ao número de itens e dimensões do instrumento (Arrindell, 1980; Arrindell et al., 2003; Arrindell, Emmelkamp, \& van der Ende, 1984; Arrindell \& van der Ende, 1986; Beck, Carmin, \& Henninger, 1998; de Aldaz, 1982; Mellon, 2000). Na década de 90, Beck e colaboradores (1998) utilizaram o FSS III de 76 itens e apuraram quatro dimensões, nomeadamente: medos sociais; medos agorafóbicos; medo de animais ou insetos; medo de sangue e de ferimentos. Anteriormente, de Aldaz (1982), a partir do FSS III de 108 itens, desen- volveu uma versão de 64 itens, distribuídos, também, em 4 dimensões, a saber: medo de situações sociais; medo de lesões e operações cirúrgicas; medo de violência e agressão corporal; e medo de animais e insetos.

Mellon (2000) no seu estudo sobre as qualidades psicométricas da FSS-GR, versão na língua grega, constituída por 90 itens, confirmou uma estrutura de cinco fatores, designadamente: medos sociais; medo de lesões de doenças e da morte; medo de cenas agressivas; medo de animais e medos agorafóbicos. Arrindell (1980), utilizando a versão constituída por 76 itens (Wolpe \& Lang, 1964), verificou que 52 desses itens se distribuíam em 5 dimensões (ansiedade social; agorafobia; medo de ferimentos, da morte e de doenças; medo de presenciar cenas agressivas e sexuais e medo de animais inofensivos). Essa estrutura fatorial, foi confirmada em diversos trabalhos (Arrindell et al., 2003; Arrindell et al., 1984; Arrindell \& van der Ende, 1986).

Diferentes populações têm sido alvo de estudo com o FSS como a população clínica (com psicopatologia), a estudantil (composta apenas por estudantes) e com menor incidência a normativa (população de toda a comunidade). Tasto (1977), em sua revisão sobre a utilização do FSS, apresenta estudos que contemplam apenas populações clínicas e estudantis, uma vez que, de acordo com o autor, não há relatos de análises fatoriais do FSS na população normativa, apontando a necessidade de estudos com essa população. Arrindell e colaboradores (1984) após verificarem a existência de apenas um estudo com população normativa, realizaram um trabalho no qual participaram indivíduos com fobias, pacientes psiquiátricos em ambulatório, estudantes e os cônjuges de pacientes psiquiátricos (que representaram a população normativa). Os autores confirmaram a estrutura fatorial da FSS III (Arrindell, 1980) e verificaram elevados coeficientes de $\alpha$ para as subescalas e para a escala geral nas diferentes amostras do estudo. Os resultados apontaram uma tendência para o aumento das correlações entre as dimensões da escala nas amostras estudantil e normativa. Mellon (2000) confirmou no seu trabalho elevados coeficientes de $\alpha$ para as subescalas e para a escala geral. Alguns itens apresentaram valores baixos de confiabilidade teste-reteste.

O FSS III tem sido utilizado em diversos países, tais como Holanda (Arrindell, 1980; Arrindell et al., 1984), Venezuela (de Aldaz, 1982), Canadá (Arrindell \& van der Ende, 1986), Estados Unidos da América (Beck et al., 1998) e Grécia (Mellon, 2000). Arrindell e colaboradores (2003) realizaram um estudo com populações de 11 países (Austrália, Alemanha Oriental, Grécia, Guatemala, Hungria, Itália, Japão, Espanha, Suécia, Reino Unido, e Venezuela).

$\mathrm{Na}$ literatura não foi encontrada uma versão do FSS na língua portuguesa, nem aplicações da FSS em Portugal ou Brasil. Assim, atendendo à importância do estudo dos medos, uma vez que o mesmo é considerado um dos maiores precursores normais no desenvolvimento da ansiedade 
Dias, F. V., Leal, I. \& Marôco, J. (2015). Inventário de Medos III: Avaliação das Qualidades Psicométricas em uma Amostra Portuguesa.

psicológica (Blanchard \& Blanchard, 1988), realizou-se este trabalho com objetivo de estimar a confiabilidade e a validade do FSS III (Arrindell, 1980), adaptado transculturalmente, para uma população normativa de língua Portuguesa.

\section{Método}

\section{Participantes}

Participaram 1.980 voluntários adultos portugueses, recrutados por conveniência de uma população normativa dos Açores, especificamente da ilha Terceira. Dos participantes, 1.172 eram mulheres, com idade compreendida entre 18 e 80 anos $(M=39,5 ; D P=8,46)$. A maioria dos sujeitos era casada $(78,3 \%)$ e tinha escolaridade máxima o $4^{\circ}$ ano do primeiro ciclo do ensino básico $(58,1 \%)$.

\section{Procedimento}

O pedido de participação foi realizado por carta dirigida ao adulto participante. Neste processo, contou-se com a colaboração de quatro escolas da ilha e respectivos alunos. Os professores de cada turma pediram aos alunos sua colaboração para um estudo. A colaboração de cada aluno consistiu em levar para casa e entregar a dois adultos do seu meio, dois questionários e uma carta de apresentação do estudo a ser desenvolvido. Na carta constavam informações relativas ao estudo e ao investigador responsável pelo projeto, incluindo os seus contactos, caso houvesse alguma dúvida por parte dos participantes. Para cada estudante, foi entregue um envelope contendo um questionário. Foi dada a instrução, por escrito de que o envelope deveria ser lacrado pelos participantes, após o seu preenchimento, no sentido de salvaguardar seu anonimato. Neste processo foram entregues 3.550 envelopes e foram devolvidos 2.020 (Taxa de resposta de 57\%). Dos questionários entregues, 40 não foram considerados válidos devido a falhas no seu preenchimento.

\section{Instrumentos}

Um questionário sociodemográfico foi elaborado para caracterização da amostra. Foram consideradas informações relativas ao sexo, idade, estado civil e nível de escolaridade. Para a avaliação dos medos utilizou-se a versão do Fear Survey Schedule III, proposta por Arrindell (1980), constituída por 52 itens, dispostos em escala do tipo Likert de 5 pontos, variando de 0 (nenhum medo) a 4 (muito medo), distribuídos em 5 dimensões (Ansiedade Social -13 itens; Agorafobia -13 itens; Medo de Ferimentos, da Morte e de Doenças -12 itens); Medo de Presenciar Cenas Agressivas e Sexuais -8 itens; e Medo de Animais Inofensivos -6 itens). A confiabilidade apresentada na versão original (Arrindell, 1980) foi de 0,95 para a escala total e relativamente às dimensões os coeficientes $\alpha$ de Cronbach foram de 0,91 para a Ansiedade Social, 0,88 para Agorafobia, 0,89 para Medo de Ferimentos, da Morte e de Doenças, 0,79 para Medo de Presenciar Cenas Agressivas e Sexuais e 0,85 para Medo de Animais Inofensivos.
Foi realizada a tradução e retradução do FSS III, a partir da versão em inglês (Arrindell et al., 2003). Participaram neste procedimento dois psicólogos com domínio da língua inglesa, que asseguraram a equivalência cultural dos enunciados. Este trabalho foi realizado em duas fases. Na primeira, foi realizada tradução dos enunciados para a língua portuguesa de Portugal por um dos psicólogos. $\mathrm{Na}$ segunda, foi realizada a retroversão pelo outro psicólogo, que não teve acesso à versão original. As versões foram analisadas por outros dois psicólogos, que não participaram no processo de tradução e retroversão, que aceitaram a tradução para a língua portuguesa de Portugal.

\section{Análise dos Dados}

A sensibilidade psicométrica dos itens foi avaliada por meio dos coeficientes de assimetria $(S K)$ e de achatamento $(K U)$. Considerou-se que coeficientes de assimetria superiores a 3 e de achatamento superiores a 7, em valor absoluto, indicavam itens com problemas de desvio significativo da normalidade que recomendam a não utilização desses itens em análises subsequentes (Kline, 1998). Realizou-se o estudo da existência de outliers por meio da análise da distância quadrada de Mahalanobis $\left(\mathrm{D}^{2}\right)$ (Hair, Black, Babin, Anderson, \& Tatham, 2005; Marôco, 2010).

Foi conduzida análise fatorial confirmatória (AFC) com método de estimação por Máxima Verosimilhança da versão portuguesa do FSS-III para verificar o grau com que as dimensões encontradas satisfaziam a estrutura fatorial original composta por cinco fatores. Utilizou-se os índices de qualidade de ajustamento $\chi^{2} / g l$ (razão qui-quadrado e graus de liberdade), CFI (Comparative Fit Index), GFI (Goodness of Fit Index), SRMR (Standardized Root Mean Square Residual) e RMSEA (Root Mean Square Error of Approximation) (Kline, 1998; Marôco, 2010). O ajustamento do modelo foi considerado bom quando os valores de CFI e GFI foram superiores a 0,9 , de SRMR foram inferiores a 0,08 e de RMSEA inferiores a 0,05 (Kline, 1998; Marôco, 2010). Para a realização da AFC foi utilizado o programa AMOS $^{\circledR} 18.0$ (SPSS Inc., Chicago, IL), com o método da máxima verossimilhança.

A amostra do estudo foi dividida aleatoriamente em duas partes denominadas "amostra de teste" $(n=1,000) \mathrm{e}$ "amostra de validação" $(n=980)$. O refinamento do modelo de medida foi efetuado na amostra de teste. Os itens que apresentaram problemas de sensibilidade foram removidos, assim como os itens com pesos fatoriais inferiores a 0,45 e/ou os que apresentaram fatores específicos (erros) correlacionados inter-fatores. Após estas modificações do modelo, sustentadas na reavaliação da importância teórica dos itens e respectivos construtos, verificou-se a adequação da estrutura fatorial proposta à amostra de teste. $O$ teste de diferenças do $\chi^{2}$ foi realizado para avaliar se o ajustamento do modelo modificado era significativamente melhor que o modelo original.

Com o objetivo de verificar a estabilidade da solução fatorial obtida, realizou-se a validação cruzada do modelo, visando comparar os índices observados na amostra teste 
com os da amostra de validação (Byrne, 2001; Marôco, 2010). A invariância do modelo de medida foi avaliada nas duas amostras por comparação do modelo livre (com pesos fatoriais e variâncias/covariâncias dos fatores livres) com um modelo constrito, onde foram fixados, sequencialmente, os pesos fatoriais e as variâncias/covariâncias das duas amostras (Byrne, 2001; Marôco, 2010). O teste da diferença de $\chi^{2}\left(\Delta \chi^{2}\right)$ foi usado para avaliar a significância das diferenças dos modelos ajustados às amostras de teste e de validação.

Estimou-se a confiabilidade composta (CC) e a variância extraída média (VEM) para analisar a validade convergente de cada fator (Fornell \& Larcker, 1981; Marôco, 2010). De acordo com Hair et al. (2005), valores de $\mathrm{VEM}_{\mathrm{j}} \geq 0,5$ e $\mathrm{CC}_{\mathrm{j}} \geq 0,7$ indicam uma adequada validade convergente e $\mathrm{CC}_{\mathrm{j}}>0,7$ é indicador de boa confiabilidade. A validade discriminante foi avaliada pela comparação das VEM de cada fator com o quadrado da correlação entre os fatores. Se VEM $\mathrm{e} V E M_{\mathrm{i}} \geq \rho_{\mathrm{ij}}{ }^{2}$ existe evidência de validade discriminante (Fornell \& Larcker, 1981; Marôco, 2010).
A confiabilidade foi analisada por meio do coeficiente $\alpha$ de Cronbach padronizado e estratificado (Cronbach, 1951; Marôco \& Garcia-Marques, 2006). Considerou-se que os fatores apresentaram confiabilidade adequada quando $\alpha>$ 0,7 (Marôco \& Garcia-Marques, 2006).

Após análise das correlações e da validade discriminante entre os fatores, propôs-se uma estrutura hierárquica com um fator latente global de $2^{\mathrm{a}}$ ordem, denominado "Medos". A qualidade do modelo hierárquico de segunda ordem foi avaliada em relação ao modelo com estrutura fatorial de $1^{\mathrm{a}}$ ordem.

\section{Resultados}

Validade Fatorial do Modelo de Medida Original

A Tabela 1 apresenta as estatísticas referentes à sensibilidade psicométrica dos itens (média, mediana, moda, desvio-padrão, curtose, assimetria). Os itens 17, 18, 19, $24,31,48$ e 49 foram removidos, por apresentarem desvios severos à normalidade que não recomendam o seu uso na análise fatorial subsequente.

Tabela 1

Sensibilidade Psicométrica dos Itens do FSS III

\begin{tabular}{ccccccc}
\hline Itens FSS III & Média & Mediana & Moda & Desvio-padrão & Curtose & Assimetria \\
\hline it1 & 1,25 & 1 & 0 & 1,22 & $-0,57$ & 0,63 \\
it2 & 0,65 & 0 & 0 & 1,05 & 1,90 & 1,64 \\
it3 & 1,12 & 1 & 0 & 1,13 & $-0,22$ & 0,77 \\
it4 & 1,18 & 1 & 0 & 1,35 & $-0,48$ & 0,86 \\
it5 & 1,08 & 1 & 0 & 1,11 & $-0,38$ & 0,70 \\
it6 & 0,62 & 0 & 0 & 0,98 & 1,75 & 1,57 \\
it7 & 1,48 & 1 & 0 & 1,24 & $-0,72$ & 0,47 \\
it8 & 1,15 & 1 & 0 & 1,21 & $-0,30$ & 0,81 \\
it9 & 1,50 & 1 & 0 & 1,28 & $-0,93$ & 0,38 \\
it10 & 0,59 & 0 & 0 & 0,87 & 1,84 & 1,47 \\
it11 & 1,74 & 2 & 0 & 1,43 & $-1,23$ & 0,27 \\
it12 & 0,66 & 0 & 0 & 0,97 & 1,68 & 1,49 \\
it13 & 1,40 & 1 & 0 & 1,29 & $-0,75$ & 0,56 \\
it14 & 0,84 & 0 & 0 & 1,23 & 0,70 & 1,36 \\
it15 & 0,92 & 1 & 0 & 1,05 & 0,18 & 0,95 \\
it16 & 1,30 & 1 & 0 & 1,36 & $-0,82$ & 0,68 \\
it17 & 0,36 & 0 & 0 & 0,82 & 7,09 & 2,66 \\
it18 & 0,17 & 0 & 0 & 0,53 & 16,70 & 3,79 \\
it19 & 0,13 & 0 & 0 & 0,43 & 18,15 & 3,95 \\
it20 & 0,38 & 0 & 0 & 0,75 & 5,66 & 2,30 \\
it21 & 0,85 & 0 & 0 & 1,08 & 0,76 & 1,22 \\
it22 & 0,92 & 0 & 0 & 1,23 & 0,25 & 1,17
\end{tabular}


Dias, F. V., Leal, I. \& Marôco, J. (2015). Inventário de Medos III: Avaliação das Qualidades Psicométricas em uma Amostra Portuguesa.

\begin{tabular}{|c|c|c|c|c|c|c|}
\hline it23 & 0,55 & 0 & 0 & 0,94 & 2,92 & 1,83 \\
\hline it24 & 0,29 & 0 & 0 & 0,70 & 8,31 & 2,80 \\
\hline it25 & 0,77 & 0 & 0 & 0,99 & 0,77 & 1,18 \\
\hline it26 & 0,78 & 0 & 0 & 0,97 & 0,79 & 1,16 \\
\hline it27 & 0,60 & 0 & 0 & 0,93 & 2,63 & 1,71 \\
\hline it 28 & 1,09 & 1 & 0 & 1,33 & $-0,28$ & 0,98 \\
\hline it29 & 1,50 & 1 & 0 & 1,45 & 0,63 & 0,73 \\
\hline it30 & 1,19 & 1 & 0 & 1,35 & 4,41 & 1,35 \\
\hline it31 & 0,34 & 0 & 0 & 0,78 & 7,06 & 2,64 \\
\hline it32 & 0,55 & 0 & 0 & 0,90 & 2,97 & 1,78 \\
\hline it33 & 0,86 & 1 & 0 & 1,05 & 0,50 & 1,11 \\
\hline it34 & 0,81 & 0 & 0 & 1,01 & 0,62 & 1,14 \\
\hline it35 & 0,91 & 0 & 0 & 1,29 & 0.29 & 1,24 \\
\hline it36 & 1,91 & 2 & 0 & 1,45 & $-1,32$ & 0,06 \\
\hline it37 & 1,78 & 2 & 0 & 1,54 & $-1,46$ & 0,19 \\
\hline it38 & 0,96 & 0 & 0 & 1,20 & $-0,06$ & 1,02 \\
\hline it39 & 0,73 & 0 & 0 & 1,07 & 1,01 & 1,38 \\
\hline it40 & 1,10 & 1 & 0 & 1,30 & $-0,36$ & 0,92 \\
\hline it41 & 1,27 & 1 & 0 & 1,19 & $-0,48$ & 0,62 \\
\hline it42 & 0,99 & 0 & 0 & 1,31 & 1,23 & 1,26 \\
\hline it43 & 0,79 & 0 & 0 & 1,09 & 0,97 & 1,32 \\
\hline it44 & 1,22 & 1 & 0 & 1,12 & $-0,31$ & 0,65 \\
\hline it45 & 2,04 & 2 & 4 & 1,51 & $-1,43$ & $-0,06$ \\
\hline it46 & 1,06 & 0 & 0 & 1,33 & $-0,27$ & 1,00 \\
\hline it47 & 1,00 & 1 & 0 & 1,15 & 0,04 & 0,97 \\
\hline it48 & 0,25 & 0 & 0 & 0,73 & 12,07 & 3,44 \\
\hline it49 & 0,18 & 0 & 0 & 0,61 & 19,30 & 4,23 \\
\hline it50 & 0,41 & 0 & 0 & 0,89 & 5,44 & 2,40 \\
\hline it51 & 1,60 & 2 & 2 & 1,22 & $-0,66$ & 0,36 \\
\hline it52 & 1,49 & 1 & 0 & 1,28 & $-0,76$ & 0,46 \\
\hline
\end{tabular}

A análise fatorial confirmatória do FSS-III com 5 fatores, na amostra global, indicou que o modelo original proposto por Arrindell (1980) apresentava um ajustamento inaceitável, $\chi^{2} / g l=8,149 ; \mathrm{CFI}=0,77, \mathrm{GFI}=0,82$, RMSEA $=0,06 ; P($ rmsea $)<0,001$. Assim, procedeu-se ao refinamento do modelo como descrito na seção dos métodos.

O refinamento do modelo de medida foi efetuado na amostra de teste e o ajustamento do modelo original foi considerado inaceitável, $\chi^{2} / g l=4,830 ; \mathrm{CFI}=0,75, \mathrm{GFI}=$ 0,79, SRMR $=0,07$, RMSEA $=0,06 ; P($ rmsea $)<0,001$. Em um primeiro passo, os itens 10, 20, 32 e 50 foram removidos por apresentarem pesos fatoriais inferiores a 0,45 .
Posteriormente, foram removidos os itens 2, 35, 39, $46 \mathrm{e}$ 51, uma vez que os seus índices de modificação sugeriram a correlação dos respectivos erros de medida intra-fatores. Os itens 7, 15 e 43 foram removidos por apresentarem índices de modificação que sugeriram a correlação dos respectivos erros de medida inter-fatores.

A remoção dos itens, referidos anteriormente, permitiu obter uma boa qualidade de ajustamento fatorial, $\chi^{2} / g l=$ 3,$249 ; \mathrm{CFI}=0,90, \mathrm{GFI}=0,91, \mathrm{SRMR}=0,06, \mathrm{RMSEA}=$ 0,$05 ; P(\mathrm{rmsea})=0,945$, atestando a validade fatorial do FSS III. O modelo simplificado apresentou uma qualidade de ajustamento significativamente superior ao modelo original, $\Delta \mathrm{X}^{2}(779)=4528941, p<0,05$. 


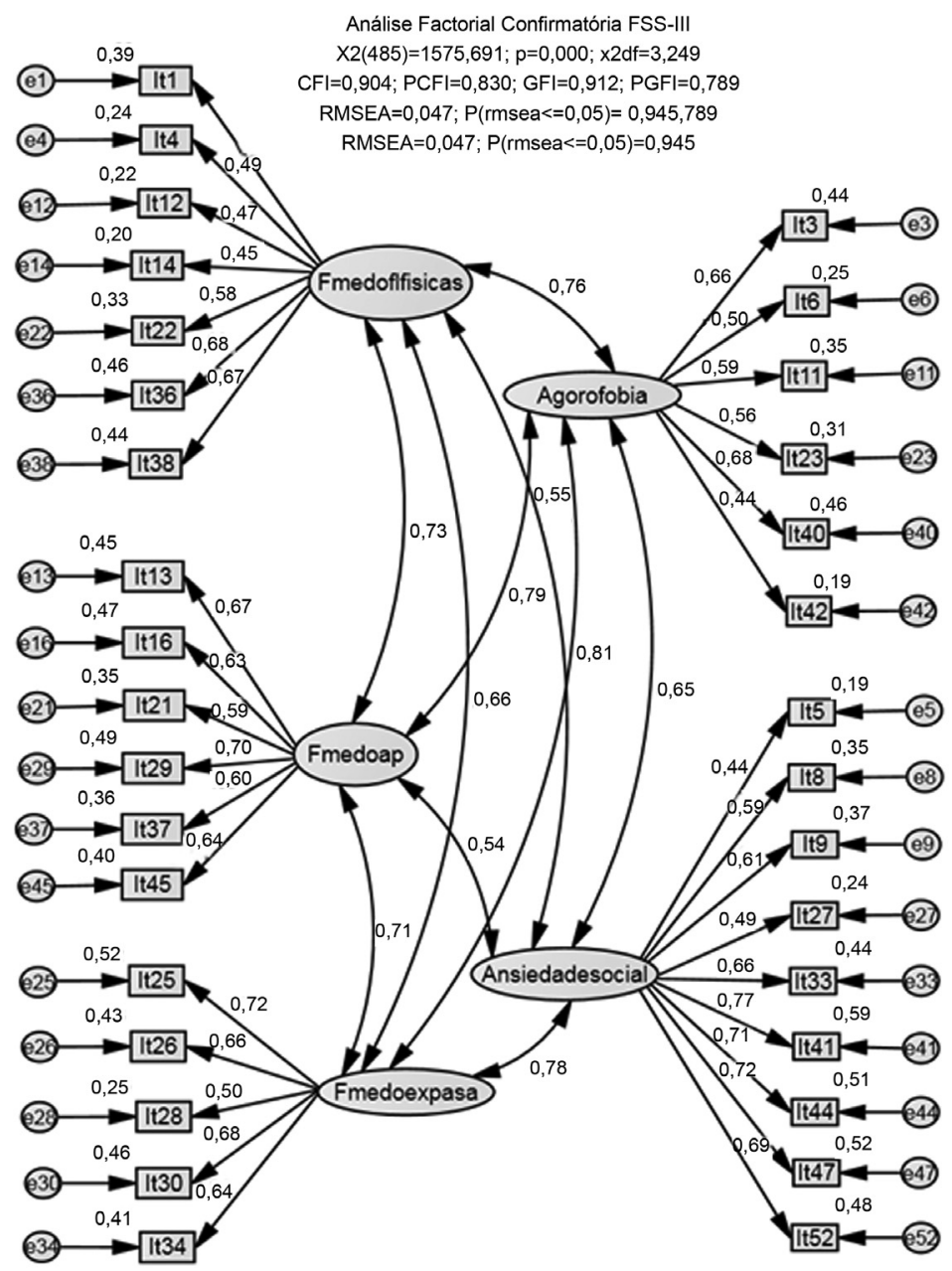

Figura 1. Modelo ajustado.

Análise de Invariância do Modelo de Medida Ajustado

O modelo fatorial apresentou um bom ajustamento, simultaneamente à amostra de teste e à amostra de validação, $\chi^{2}(970)=3431,7 ; p<0,001 ; \chi^{2} / g l=3,5 ; \mathrm{CFI}=0,9 \mathrm{GFI}=$ 0,9 , RMSEA $=0,04 ; P($ rmsea $)=1,000$. Os resultados da comparação do modelo livre com um modelo constrito, onde foram fixados os pesos fatoriais e as variâncias/ covariâncias das duas amostras, atestaram a invariância do modelo fatorial para a amostra de teste e amostra de validação, Pesos fatoriais: $\Delta \chi^{2}(33)=29,2 ; p=0,658$; Covariâncias: $\Delta \chi^{2}(10)=20,4 ; p=0,026$.

\section{Confiabilidade, Validade Convergente e Discriminante do} Modelo de Medida Ajustado

Os valores de consistência interna $(\alpha)$, confiabilidade composta $(C C)$, variância extraída da média (VEM) e quadrado da correlação entre fatores $\left(\rho^{2}\right)$ estão apresentados na Tabela 2.

A consistência interna e a confiabilidade composta foram adequadas para todos os fatores. $\mathrm{Na}$ amostra de teste, obteve-se baixos valores da VEM. Apenas os fatores Medo de ferimentos, da morte e de doenças e Ansiedade social, e os fatores Medo de animais inofensivos e
Ansiedade social, apresentaram evidências de validade discriminante.

\section{Análise Fatorial de $2^{a}$ Ordem}

Uma vez que se verificaram correlações elevadas e estatisticamente significativas entre os 5 fatores de $1^{\text {a }}$ ordem (Figura 1), foi elaborado um modelo com fator de $2^{\mathrm{a}}$ ordem, que foi denominado por "Medos". Este procedimento baseou-se não apenas na questão estatística, que defende a existência de um fator de $2^{\mathrm{a}}$ ordem quando se verificam fortes relações entre os fatores de $1^{\mathrm{a}}$ ordem (Bollen, 1989; Marôco, 2010), como também considerou o modelo teórico de Taylor (1998) e a diferente contribuição das diferentes dimensões dos medos para a análise de um fator geral (Arrindell, 1980).

A Figura 2 apresenta o modelo do FSS III modificado com o fator de $2^{\mathrm{a}}$ ordem. Neste modelo, os valores de qualidade de ajustamento foram considerados bons, $\chi^{2} / g l$ $=3,4 ; \mathrm{CFI}=0,9 \mathrm{GFI}=0,9, \mathrm{RMSEA}=0,049 ; P(\mathrm{rmsea})$ $=0,729$. Todas as trajetórias entre o fator de $2^{\mathrm{a}}$ ordem e os fatores de $1^{\text {a }}$ ordem foram estatisticamente significativas $(p<0,001)$. Recorrendo aos pesos dos escores fatoriais calculados pelo AMOS, foi possível estimar o 
Escore global de "Medos" (valores padronizados) com a expressão:

"Medos" $=0,042 I t_{13}+0,042 I t_{16}+0,037 I t_{21}+0,042 I t_{29}$

$+0,026 I t_{37}+0,031 I_{45}+0,099 I_{25}+0,081 I_{26}+0,033 I t_{28}$

$$
\begin{aligned}
& +0,065 I t_{30}+0,072 I t_{34}+0,036 I t_{1}+0,021 I t_{4}+0,028 I t_{12} \\
& +0,020 I t_{14}+0,032 I t_{22}+0,039 I t_{36}+0,044 I t_{38}+0,024 I t_{52} \\
& +0,030 I t_{47}+0,029 I t_{44}+0,037 I t_{41}+0,025 I t_{33}+0,016 I t_{27} \\
& +0,017 I t_{9}+0,017 I t_{8}+0,0111 t_{5}+0,034 I t_{42}+0,078 I t_{40}+ \\
& 0,070 I t_{23}+0,051 I t_{11}+0,055 I t_{6}+0,083 I t_{3}
\end{aligned}
$$

Tabela 2

FSS III - Análise da Consistência Interna ( $\alpha$ ), da Confiabilidade Composta (CC), da Variância Extraída Média (VEM) e do Quadrado da Correlação entre Fatores ( $\left.\rho^{2}\right)$ do Modelo de Medida Ajustado

\begin{tabular}{lcccc}
\hline Fatores & $\alpha$ & CC & VEM & $\rho^{2}$ \\
\hline Ansiedade Social & 0,858 & 0,848 & 0,409 & $0,29-0,61$ \\
Agorafobia & 0,727 & 0,747 & 0,335 & $0,42-0,66$ \\
Medo de Ferimentos da Morte ou de Doenças & 0,779 & 0,769 & 0,328 & $0,30-0,58$ \\
Medo de Presenciar Cenas Agressivas e Sexuais & 0,766 & 0,777 & 0,414 & $0,44-0,66$ \\
Medo de Animais Inofensivos & 0,812 & 0,812 & 0,419 & $0,29-0,62$ \\
Escala $\alpha_{\text {estratificado }}$ & 0,944 & & & \\
\hline
\end{tabular}

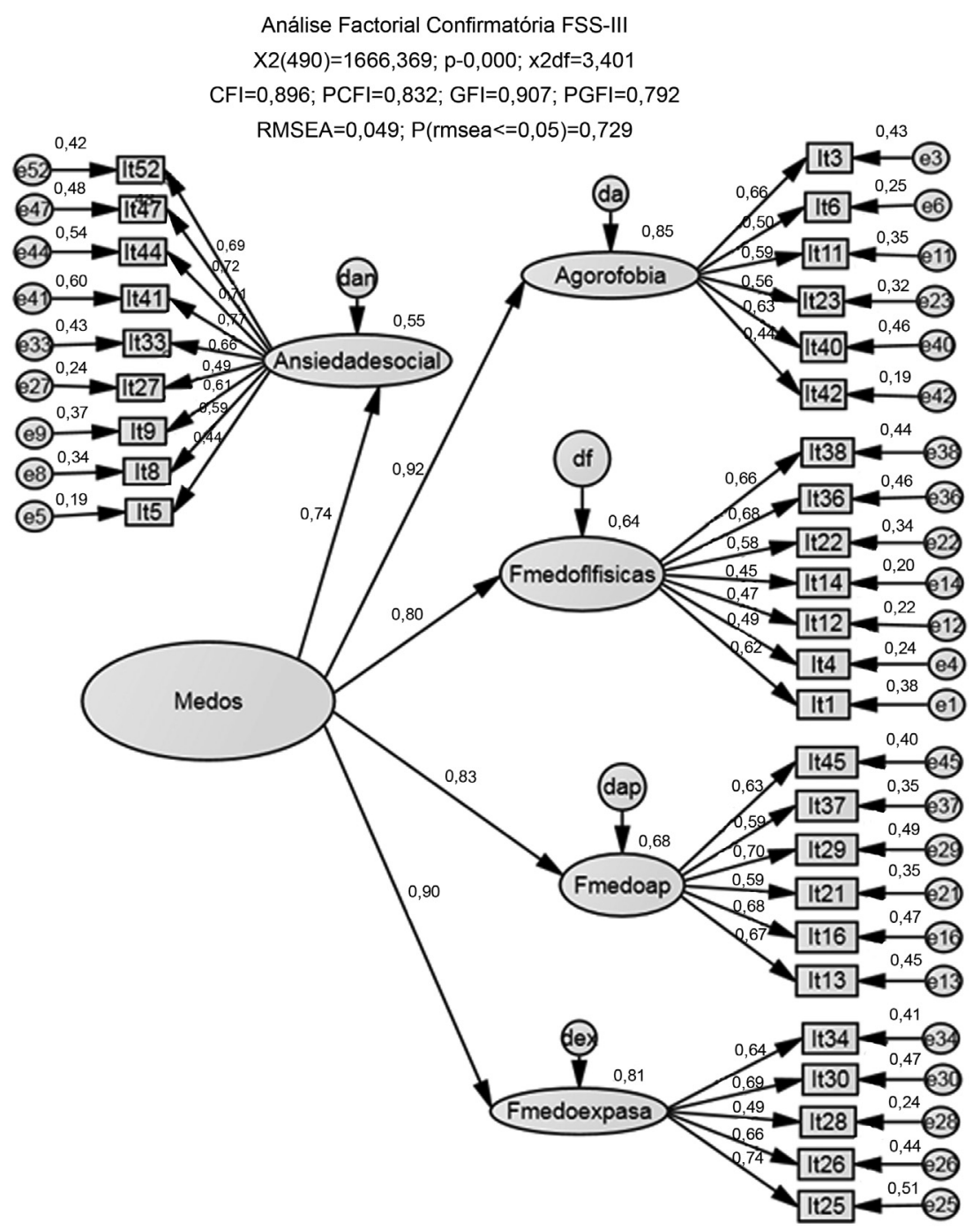

Figura 2. Modelo modificado com o fator de $2^{\mathrm{a}}$ ordem. 


\section{Discussão}

A estrutura fatorial do FSS III (Arrindell, 1980) foi confirmada na amostra portuguesa, após redução dos itens que se apresentaram problemáticos. A estrutura proposta manteve-se invariante em uma amostra independente advinda da mesma população.

Apesar desta estrutura fatorial apresentar-se estável em diversos estudos mantendo os 52 itens (Arrindell, 1980; Arrindell et al., 2003; Arrindell et al., 1984; Arrindell \& van der Ende, 1986), no presente trabalho foi necessária a remoção de alguns itens da escala original. Esta decisão foi tomada com objetivo de produzir uma escala com maior confiabilidade e validade para a população em estudo.

Como refere Mellon (2000) o cálculo da validade fatorial é fundamental para verificar se a especificação dos itens de um determinado construto é correta, e baseia-se na avaliação dos pesos fatoriais padronizados. No entanto, os valores considerados aceitáveis não são consensuais na literatura (Arrindell et al., 2003). Atendendo às sugestões de Marôco (2010) relativas aos valores dos pesos fatoriais e às advertências de Hersen (1973) sobre as qualidades psicométricas do FSS, optou-se por considerar valores superiores a 0,45 como admissíveis para cada item, no entanto, outros autores (por exemplo, Arrindell et al., 2003; de Aldaz, 1982; Mellon, 2000) consideraram valores inferiores a 0,45. Em seu estudo, Arrindell e colaboradores (2003) optaram pela manutenção de itens cujos pesos fatoriais apresentaram valores superiores ou iguais a 0,31 . Os itens 7, 17 e 31, removidos no presente trabalho, apresentaram no estudo dos referidos autores pesos fatoriais inferiores a 0,40. Nesse mesmo estudo, outros itens, não identificados, foram aceitos mesmo apresentando valores entre 0,40 e 0,45 . Assim, entende-se que os diferentes valores referenciais admitidos influenciaram diretamente a remoção ou manutenção dos itens. $\mathrm{O}$ baixo peso fatorial apresentado por alguns itens pode estar relacionado às palavras que o compõem o que pode gerar diferença de interpretação e conotação, dependendo do sujeito que o analisa (Arrindell et al., 1984). Este fato pode ser ainda mais agravado pelas diferentes condições sócio-culturais observadas entre a população holandesa (do estudo original) e a população portuguesa (do presente estudo). Beaton, Bombardier, Guillemin, e Ferraz, (2000) alertaram que essas diferenças sempre ocorrem na adaptação transcultural de um instrumento psicométrico. Outro aspecto a ser destacado é a disparidade entre o tipo de amostra empregada em diferentes estudos, o que pode levar a conotação distinta dos itens quando o mesmo é lido por indivíduos pertencentes a amostras clínicas, estudantis ou de pessoas adultas. De salientar as diferentes características (por exemplo, idade, nível de escolaridade, etapa do ciclo de vida e vivências do dia a dia) dos sujeitos que participaram nos diversos trabalhos aqui apresentados (Arrindell, 1980; Arrindell et al., 2003; Arrindell et al., 1984; Arrindell \& van der Ende, 1986). Se tomarmos os conceitos de medo e ansiedade descritos inicialmente (Öhman, 2008), podemos colocar a hipótese de que as diferentes características das populações, e relativas diferenças na avaliação e percepção de ameaça, têm reflexo na interpretação do item, influenciando consequentemente o peso que cada item apresenta para cada dimensão, bem como para o inventário total. Embora o estudo de Arrindell e colaboradores (1984) tenha contado com uma amostra não clínica/não estudantil, a mesma era constituída pelos parceiros de pacientes em ambulatório, possivelmente mais familiarizados, pelas suas vivências, com os conteúdos dos itens, permitindo uma conotação semelhante à verificada com as amostras clínicas.

A versão da FSS III em português mostrou adequada consistência interna e confiabilidade composta (Tabela 2). Estes resultados são consonantes com a literatura (Arrindell, 1980; Arrindell et al., 2003; Arrindell et al., 1984; Arrindell \& van der Ende, 1986; Beck et al., 1998; de Aldaz, 1982; Mellon, 2000). No presente trabalho os itens de teor sexual não apresentaram peso fatorial considerável e por esse motivo foram removidos o que pode dever-se às características da nossa amostra. Arrindell e colaboradores (1984) verificaram, para esta dimensão, coeficientes alfa de Cronbach mais baixos que os verificados nas amostras estudantis $(0,60-0,67)$. Estes resultados podem sugerir que amostras não clínicas percebem certos estímulos (por exemplo mulheres e/ou homens nus) de forma menos ameaçadora comparativamente à população clínica.

As questões da validade são mais difíceis de serem examinadas comparativamente a outros estudos da literatura, uma vez que os mesmos ou não fizeram esse tipo de análise, ou utilizaram métodos estatísticos diferentes dos realizados no presente trabalho. Nenhum trabalho apresentou evidências relacionadas com a validade convergente dos fatores da FSS III. Arrindell et al. (2003) não realizaram a análise da validade do instrumento para as amostras em estudo, no entanto, deixaram como recomendação a sua execução em diferentes países.

A validade convergente e discriminante do FSS III foi baixa, o que pode ser atribuído às fortes correlações (Figura 1) entre as várias dimensões do instrumento, fato que sugere cautela na sua interpretação.

Diversos trabalhos têm proposto o FSS III como um instrumento com 4 ou 5 dimensões definidas (Arrindell et al., 2003; Arrindell et al., 1984; Arrindell \& van der Ende, 1986; Beck et al., 1998). A defesa principal desta ideia baseia-se no valor que a utilização desse tipo de instrumento tem na prática clínica, uma vez que, organizado em fatores é permitida uma avaliação independente dos vários tipos de medos, oferecendo informação sobre possíveis áreas a trabalhar com o paciente, em uma abordagem mais focalizada. No entanto, alguns trabalhos têm apontado uma maior correlação entre as dimensões deste instrumento, especialmente nas amostras não clínicas e estudantis (por exemplo, Arrindell et al., 1984) denotando uma propensão para o aumento das correlações entre as dimensões quando a patologia, nomeadamente a fobia, é menor ou inexistente (Arrindell et al., 1984). Os resultados do presente estudo confirmam e acentuam essa tendência. 
Atendendo aos resultados mencionados, ao modelo teórico dos medos proposto por Taylor (1998), assim como à hipótese de Arrindell (1980) sobre a diferente contribuição dos diversos medos para um escore global, propomos um fator de segunda ordem, denominado de "Medos", que visa caracterizar o indivíduo em relação à intensidade e prevalência dos variados Medos (medos sociais; medo de lesões de doenças e da morte; medo de cenas agressivas; medo de animais; e medos agorafóbicos). Assim, a utilização em contexto clínico, do escore obtido pelo fator de segunda ordem, daria uma visão geral e rápida dos medos do paciente, sendo o uso dessa informação uma importante ferramenta no delineamento da intervenção a ser realizada (de Jongh et al., 2011).

Futuras investigações que contemplem uma análise das vivências dos indivíduos, sua percepção de ameaça e consequente reflexo na análise da prevalência e intensidade dos seus medos, com recurso ao inventário FSS III, poderiam fornecer dados importantes para o estudo dos medos nas populações normativas e consequente trabalho de prevenção da patologia. A ausência de resultados de outras escalas que avaliem os medos, não possibilitou a análise da validade concorrente da FSS III, sendo uma limitação deste estudo que deverá ser avaliada em trabalhos futuros. É porém de referir que a FSS III é a escala atualmente mais usada na avaliação de medos, sendo esta a utilizada como padrão-ouro em estudos desta área.

\section{Conclusão}

A estrutura original do FSS III foi confirmada neste trabalho, após a redução dos itens, e manteve-se invariante em uma amostra independente advinda da mesma população e mostrou adequada confiabilidade. Contudo, esta estrutura fatorial levantou questões quanto às validades convergente e discriminante, o que nos levou a sugerir a existência de um fator de $2^{\mathrm{a}}$ ordem que pode ser utilizado para produzir um escore global de "medos".

Este fator de $2^{\mathrm{a}}$ ordem pode ser utilizado em contexto de avaliação, para estimar uma medida de "medos" para a população portuguesa. Esta avaliação é importante nos campos da prevenção e intervenção, uma vez que os medos são considerados um dos maiores precursores do desenvolvimento das patologias da ansiedade.

\section{Referências}

American Psychiatric Association. (2002). DSM-IV-TR: Manual de diagnóstico e estatistica das perturbações mentais (4. ed.). Lisboa, Portugal: Autor.

Arrindell, W. A. (1980). Dimensional structure and psychopathology correlates of the fear survey schedule (FSS-III) in a phobic population: A factorial definition of agoraphobia. Behavior Research and Therapy, 18, 229-242. doi:10.1016/00057967(80)90080-7

Arrindell, W. A., Eisemann, M., Richter, J., Oei, T. P. S., Caballo, V. E., van der Ende, J., ...Cultural Clinical Psychology Study Group. (2003). Phobic anxiety in 11 nations Part I:
Dimensional constancy of the five-factor model. Behaviour Research and Therapy, 41, 461-479. doi:10.1016/S00057967(02)00047-5

Arrindell, W. A., Emmelkamp, P. M., \& van der Ende, J. (1984). Phobic dimensions: I. Reliability and generalizability across samples, gender and nations. Advances in Behaviour Research \& Therapy, 6(4), 207-254. doi:10.1016/01466402(84)90001-8

Arrindell, W. A., \& van der Ende, J. (1986). Further evidence for cross-sample invariance of phobic factors: Psychiatric inpatient ratings on the fear survey schedule-III. Behavior Research and Therapy, 24(3), 289-297. doi:10.1016/00057967(86)90188-9

Baptista, A., Carvalho, M., \& Lory, F. (2005). O medo, a ansiedade e as suas perturbações. Psicologia: Revista da Associação Portuguesa Psicologia, 19(1-2), 267-277.

Beaton, D. E., Bombardier, C., Guillemin, F., \& Ferraz, M. B. (2000). Guidelines for the process of cross-cultural adaptation of self-report measures. Spine, 24(24), 3186-3191.

Beck, J. G., Carmin, C. N., \& Henninger, N. J. (1998). The utility of the Fear Survey Schedule-III: An extended replication. Journal of Anxiety Disorders, 12(3), 177-182. doi:10.1016/ S0887-6185(98)00007-3

Blanchard, D. C., \& Blanchard, R. J. (1988). Ethoexperimental approaches to the biology of emotion. Annual Review of Psychology, 39, 43-68.

Bollen, K. A. (1989). Structural equations with latent variables. Oxford, UK: John Wiley \& Sons.

Byrne, B. M. (2001). Structural equation modeling with Amos: Basic concepts, applications and programming. Mahwah, NJ: Lawrence Erlbaum Associates.

Craske, M. G. (1977). Fear and anxiety children and adolescents. Bulletin of the Menninger Clinic, 61(2), 4-32.

Cronbach, J. L. (1951). Coefficient alpha and the internal structure of tests. Psychometrika, 16(3), 297-334. doi:10.1007/ BF02310555

De Aldaz, E. G. (1982). Factor analysis of a Venezuelan Fear Survey Schedule. Behavior Research and Therapy, 20, 313332. doi:10.1016/0005-7967(82)90090-0

De Jongh, A., Oosterink, F. M. D., Kieffer, J. M., Hoogstraten, J., \& Aartman, I. H. A. (2011). The structure of common fears: Comparing three different models. The American Journal of Psychology, 124(2), 141-149. doi:10.5406/amerjpsyc.124.2.0141

Dixon, J. J., de Monchaux, C., \& Sandler, J. (1957). Patterns of anxiety: The phobias. British Journal of Medical Psychology, 30, 34-40. doi:10.1111/j.2044-8341.1957.tb01941.x

Fornell, C., \& Larcker, D. F. (1981). Evaluating structural equation models with unobservable variables and measurement error. Journal of Marketing Research, 18(1), 39-50.

Hair, J. F., Black, W. C., Babin, B., Anderson, R. E., \& Tatham, R. L. (2005). Multivariate data analysis ( $6^{\text {th }}$ ed.). Saddle River, NJ: Prentice Hall.

Hersen, M. (1973). Self-assessment of fear. Behavior Therapy, 4, 241-257. doi:10.1016/S0005-7894(73)80034-6

Kline, B. R. (1998). Software review: Software Programs for Structural Equation Modeling: Amos, EQS, and LISREL. Journal of Psychoeducational Assessment, 16(4), 343-364.

Marôco, J. (2010). Análise de equações estruturais. Lisboa, Portugal: ReportNumber.

Marôco, J., \& Garcia-Marques, T. (2006). Qual a fiabilidade do alfa de Cronbach? Questões antigas e soluções modernas? Laboratório Psicologia, 4, 65-90. 
Mellon, R. (2000). A Greek-Language Inventory of Fears: Psychometric properties and factor structure of self-reports of fears on the Hellenic Fear Survey Schedule. Journal of Psychopathology and Behavioral Assessment, 22(2), 123-140. doi:10.1023/A:1007532406778

Öhman, A. (2008). Fear and anxiety: Overlaps and dissociations. In M. Lewis, J. M. Haviland-Jones, \& L. F. Barrett (Eds.), Handbook of emotions ( $3^{\text {rd }}$ ed., pp. 709-728). New York: Guilford Press.

Power, M., \& Dalgleish, T. (2008). Cognition and emotion: From order to disorder ( $2^{\text {nd }}$ ed.). New York: Psychology Press.

Rachman, S. J. (2004). Fear and courage: A psychological perspective. Social Research, 71(1), 149-176.

Rosen, J. B., \& Schulkin, J. (1998). From normal fear to pathological anxiety. Psychological Review, 105(2), 325-350. doi:10.1037/0033-295X.105.2.325

Sylvers, P., Lilienfeld, S. O., \& LaPrairie, J. L. (2011). Differences between trait fear and trait anxiety: Implications for psychopathology. Clinical Psychology Review, 31(1), 122137. doi:10.1016/j.cpr.2010.08.004

Tasto, D. L. (1977). Self-report schedules and inventories. In A. R. Ciminero, K. S. Calhoun, \& H. E. Adms (Eds.), Handbook of behavioral assessment (pp. 153-193). New York: WileyInterscience.

Taylor, S. (1998). The hierarchic structure of fears. Behaviour Research \& Therapy, 36(2), 205. doi:10.1016/S00057967(98)00012-6

Wolpe, J., \& Lang, P. J. (1964). A fear survey schedule for use in behaviour therapy. Behaviour Research and Therapy, 2(1), 27-30. doi:10.1037/t13760-000

Os autores agradecem à escola Escola Básica Integrada dos Biscoitos, à Escola Básica Integrada da Praia da Vitória, à Escola Básica Integrada de Angra do Heroísmo e a Escola Básica e Secundária Tomás de Borba, nomeadamente aos diretores Dr. Luís Filipe Martinho Miranda, Dr. ${ }^{a}$ Ana Vitória Silvestre Campina Bettencourt Rodrigues, Dr. João Carlos Amador Lopes Mateus, Dr. Augusto Fernando Silva Oliveira, aos docentes e funcionários envolvidos no processo, pela ajuda prestada na recolha de dados. A autora Filomena Valadão Dias agradece: à Professora Doutora Juliana Bonini Campos pelo seu precioso contributo na escrita do presente artigo em português do Brasil; à Msc Raquel V. Oliveira pela sua ajuda em diferentes fases do trabalho. A autora também agradece à Fundação Portuguesa para a Ciência e Tecnologia (FCT), pela bolsa atribuída SFRH/BD/63377/2009. 